\title{
Fixed Point Results for K-Iteration Using Non-Linear Type Mappings
}

\author{
Anju Panwar1, Ravi Parkash Bhokal ${ }^{2 *}$ \\ ${ }^{1}$ Department of Mathematics, M. D. U. Rohtak, Haryana, India \\ ${ }^{2}$ Government College, Dujana, Jhajjar (Haryana), India \\ Email: anjupanwar15@gmail.com, ^ravibhokal08@gmail.com
}

How to cite this paper: Panwar, A. and Bhokal, R.P. (2019) Fixed Point Results for K-Iteration Using Non-Linear Type Mappings. Open Access Library Journal, 6: e5245.

https://doi.org/10.4236/oalib.1105245

Received: February 13, 2019

Accepted: March 17, 2019

Published: March 20, 2019

Copyright () 2019 by author(s) and Open Access Library Inc.

This work is licensed under the Creative

Commons Attribution International

License (CC BY 4.0).

http://creativecommons.org/licenses/by/4.0/

c) (7) Open Access

\begin{abstract}
In this paper we establish convergence and stability results using general contractive condition, quasi-nonexpansive mapping and mean non expansive mapping for $\mathrm{K}$-iteration process. We shall also generalize the $\mathrm{K}$-iteration process for a pair of distinct mappings and with the help of example we claim that the generalized iteration process has better convergence rate than the $\mathrm{K}$-iteration process for single mapping and some of the existing iteration processes. Suitable examples are given in the support of main results.
\end{abstract}

\section{Subject Areas}

Mathematical Analysis

\section{Keywords}

K-Iteration Process, Opial's Condition, Mean Non-Expansive Mapping, Quasi Non-Expansive Mapping

\section{Introduction and Preliminary Definitions}

Let $(X, d)$ be a metric space and $T: X \rightarrow X$ be a self map defined on $X$. Let $F(T)=\{z \in X: T z=z\}$ denote the set of fixed point of $T$. For $x_{0} \in X$, the sequence $\left\{x_{n}\right\}_{n=0}^{\infty}$ defined by

$$
x_{n+1}=T x_{n}, n \geq 0,
$$

is called the Picard iteration.

$$
\begin{aligned}
& \text { For } x_{0} \in X \text {, the sequence }\left\{x_{n}\right\}_{n=0}^{\infty} \text { defined by } \\
& \qquad x_{n+1}=\left(1-\alpha_{n}\right) x_{n}+\alpha_{n} T x_{n}, n \geq 0,
\end{aligned}
$$

where $\left\{\alpha_{n}\right\}_{n=0}^{\infty}$ is a sequence in $[0,1]$ such that $\sum_{n=0}^{\infty} \alpha_{n}=\infty$ is called the ${ }^{\star}$ Corrosponding author. 
Mann iteration process [1].

In 2013, Khan [2] produced a new type of iteration process by introducing the concept of the following Picard-Mann hybrid iterative process for a single mapping $T$. For the initial value $x_{0} \in X$, the sequence $\left\{x_{n}\right\}_{n=0}^{\infty}$ defined by

$$
\begin{gathered}
x_{n+1}=T y_{n}, \\
y_{n}=\left(1-\alpha_{n}\right) x_{n}+\alpha_{n} T x_{n}, n \geq 0,
\end{gathered}
$$

where $\left\{\alpha_{n}\right\}_{n=0}^{\infty}$ is a sequence in $[0,1]$.

Khan [2] showed that the rate of convergence of Picard-Mann hybrid iterative process is more than the Picard iteration scheme, Mann iteration scheme [1] and Ishikawa iterative schemes [3].

In this direction Gursoy and Karakaya [4], gave new iteration process as follows:

For the initial value $x_{0} \in X$, the sequence $\left\{x_{n}\right\}_{n=0}^{\infty}$ defined by

$$
\left\{\begin{array}{l}
z_{n}=\left(1-\beta_{n}\right) x_{n}+\beta_{n} T x_{n}, \\
y_{n}=\left(1-\alpha_{n}\right) T x_{n}+\alpha_{n} T z_{n}, \\
x_{n+1}=T y_{n}
\end{array}\right.
$$

where $\left\{\alpha_{n}\right\}_{n=0}^{\infty},\left\{\beta_{n}\right\}_{n=0}^{\infty}$ is a sequence in $[0,1]$ is known as Picard-S iterative process. By giving appropriate example, Gursoy and Karakaya [4] proved that their iterative process has better convergence rate than Picard, Mann, Ishikawa, Noor and Normal-S iterative processes.

Karakaya et al. in their paper [5], introduced a new hybrid iterative process as

$$
\left\{\begin{array}{l}
x_{0} \in X, \\
y_{n}=T\left(1-\beta_{n}\right) x_{n}+\beta_{n} T x_{n}, \\
x_{n+1}=T\left(1-\alpha_{n}\right) y_{n}+\alpha_{n} T y_{n}
\end{array}\right.
$$

where $\left\{\alpha_{n}\right\}_{n=0}^{\infty},\left\{\beta_{n}\right\}_{n=0}^{\infty}$ is a sequence in $[0,1]$.

With the help of suitable example it was claimed by Karakaya et al. [5], that their iteration process converges faster than the iteration process of Gursoy and Karakaya [4].

In 2016, Thakur et al. [6] introduced a new iteration scheme called Thakur New Iteration Scheme as for the initial value $x_{0} \in X$, the sequence $\left\{x_{n}\right\}_{n=0}^{\infty}$ defined by

$$
\left\{\begin{array}{l}
z_{n}=\left(1-\beta_{n}\right) x_{n}+\beta_{n} T x_{n}, \\
y_{n}=T\left(1-\alpha_{n}\right) x_{n}+\alpha_{n} z_{n}, \\
x_{n+1}=T y_{n}
\end{array}\right.
$$

where $\left\{\alpha_{n}\right\}_{n=0}^{\infty},\left\{\beta_{n}\right\}_{n=0}^{\infty}$ is a sequence in $[0,1]$.

In [6] it was claimed that the Thakur New Iteration Scheme has higher convergence rate than the iteration process of Karakaya et al. [7].

In the recent work of Hussain et al. [8], a new iteration scheme has been developed and it is claimed that it has better convergence rate than the iterative process Thakur et al. [6]. This iteration process is called K-iteration process and is given as: 
For the initial value $x_{0} \in X$, the sequence $\left\{x_{n}\right\}_{n=0}^{\infty}$ defined by

$$
\left\{\begin{array}{l}
z_{n}=\left(1-\beta_{n}\right) x_{n}+\beta_{n} T x_{n}, \\
y_{n}=T\left(1-\alpha_{n}\right) T x_{n}+\alpha_{n} T z_{n}, \\
x_{n+1}=T y_{n}
\end{array}\right.
$$

where $\left\{\alpha_{n}\right\}_{n=0}^{\infty},\left\{\beta_{n}\right\}_{n=0}^{\infty}$ is a sequence in $[0,1]$.

In the present work we shall generalize some convergence and stability results for K-iteration process. We shall also prove convergence and stability results for more general form of $\mathrm{K}$-iteration process and $\mathrm{K}$-iteration process for a pair of two distinct mappings.

Definition 1.1 [3]: Let $X$ be a real Banach space. The mapping $T: X \rightarrow X$ is said to be asymptotically quasi-nonexpansive if $F(T) \neq \varnothing$ and there exists a sequence $\left\{\mu_{n}\right\} \subset[0, \infty)$ with $\mu_{n} \rightarrow 0$ as $n \rightarrow \infty$ such that

$$
\left\|T^{n} x-q\right\| \leq\left(1+\mu_{n}\right)\|x-q\|
$$

for all $x \in X, q \in F(T)$ and $n \geq 0$.

Definition 1.2 [9]: Let $X$ be a real Banach space. The mapping $T: X \rightarrow X$ is said to be mean non-expansive if there exists two non negative real numbers $a, b$ such that $a+b \leq 1$ and for all $x, y \in X$,

$$
\|T x-T y\|=a\|x-y\|+b\|x-T y\|
$$

Definition 1.3 [10]: Let $\left\{z_{n}\right\}_{n=0}^{\infty}$ be any sequence in $X$. Then the iterative process $x_{n+1}=f\left(T, x_{n}\right)$ which converges to a fixed point $q$, is said to be stable with respect to the mapping $T$ if for $\varphi_{n}=\left\|z_{n+1}-f\left(T, z_{n}\right)\right\|, n=0,1,2, \cdots$, we have $\lim _{n \rightarrow \infty} \varphi_{n}=0$ if and only if $\lim _{n \rightarrow \infty} z_{n}=q$.

Definition 1.4 [7]: A space $X$ is said to satisfy Opial's condition if for each sequence $\left\{x_{n}\right\}_{n=0}^{\infty}$ in $X$ such that $x_{n}$ converges weakly to $X$ we have for all $y \in X, \quad x \neq y$ following holds:

1) $\liminf _{n \rightarrow \infty}\left\|x_{n}-x\right\|<\liminf _{n \rightarrow \infty}\left\|x_{n}-y\right\|$,

2) $\limsup _{n \rightarrow \infty}\left\|x_{n}-x\right\|<\limsup _{n \rightarrow \infty}\left\|x_{n}-y\right\|$.

Lemma 1.5 [11]: Let $\left\{a_{n}\right\}_{n=0}^{\infty}$ and $\left\{b_{n}\right\}_{n=0}^{\infty}$ be non-negative real sequences satisfying the inequality:

$$
a_{n+1} \leq\left(1-b_{n}\right) a_{n}+b_{n},
$$

where $b_{n} \in(0,1)$, for all $n \in N, \sum_{n=1}^{\infty} b_{n}=\infty$ and $\frac{b_{n}}{a_{n}} \rightarrow 0$ as $n \rightarrow \infty$, then $\lim _{n \rightarrow \infty} a_{n}=0$.

Lemma 1.6 [12]: Let $\delta$ be a real number such that $0 \leq \delta<1$, and $\left\{\epsilon_{n}\right\}_{n=0}^{\infty}$ be a sequence of positive numbers such that $\lim _{n \rightarrow \infty} \epsilon_{n}=0$. Then for any sequence of positive numbers $\left\{a_{n}\right\}_{n=0}^{\infty}$ satisfying $a_{n+1} \leq \delta a_{n}+\epsilon_{n}, n=0,1,2, \cdots$, we have $\lim _{n \rightarrow \infty} a_{n}=0$.

Lemma 1.7 [13]: Let $X$ be a real Banach space and $\left\{g_{n}\right\}$ be any sequence in $X$ such that $0<g_{n}<1$ for all $n \in N$. Let $\left\{a_{n}\right\}_{n=0}^{\infty}$ and $\left\{b_{n}\right\}_{n=0}^{\infty}$ be non-negative real sequences satisfying $\limsup \left\|a_{n}\right\| \leq c, \limsup \left\|b_{n}\right\| \leq c$ and $\limsup _{n \rightarrow \infty}\left\|g_{n} a_{n}+\left(1-g_{n}\right) b_{n}\right\|=c$ holds for some ${ }^{n \rightarrow \infty} c \geq 0$. Then $\underset{n \rightarrow \infty}{\limsup }\left\|a_{n}-b_{n}\right\|=0$. 


\section{Main Results}

Theorem 2.1: Let $X$ be a Banach space and $T: X \rightarrow X$ be a mapping satisfying the condition

$$
\|T x-q\| \leq \delta\|x-q\|
$$

where $q \in F, x \in X$ and $0 \leq \delta<1$. Let $\left\{x_{n}\right\}_{n=0}^{\infty}$ be the sequence defined by the K-iterative process given by (1.7). Then the sequence $\left\{x_{n}\right\}_{n=0}^{\infty}$ converges strongly to $q \in F(T)$.

Proof: From (1.7) and (2.1) we have,

$$
\left\|x_{n+1}-q\right\|=\left\|T y_{n}-q\right\| \leq \delta\left\|T y_{n}-q\right\|
$$

And

$$
\begin{aligned}
\left\|y_{n}-q\right\| & =\left\|T\left(\left(1-\alpha_{n}\right) T x_{n}+\alpha_{n} T z_{n}\right)-q\right\| \\
& \leq \delta\left\|\left(1-\alpha_{n}\right) T x_{n}+\alpha_{n} T z_{n}-q\right\| \\
& \leq \delta\left\|\left(1-\alpha_{n}\right)\left(T x_{n}-q\right)+\alpha_{n}\left(T z_{n}-q\right)\right\| \\
& \leq \delta\left[\left(1-\alpha_{n}\right)\left\|T x_{n}-q\right\|+\alpha_{n}\left\|T z_{n}-q\right\|\right] \\
& \leq \delta\left[\left(1-\alpha_{n}\right)\left\|T x_{n}-q\right\|+\alpha_{n}\left\|T z_{n}-q\right\|\right] \\
& \leq \delta^{2}\left[\left(1-\alpha_{n}\right)\left\|x_{n}-q\right\|+\alpha_{n}\left\|z_{n}-q\right\|\right]
\end{aligned}
$$

Again using (1.7) and (2.1) we get,

$$
\begin{aligned}
\left\|z_{n}-q\right\| & =\left\|\left(1-\beta_{n}\right) x_{n}+\beta_{n} T x_{n}-q\right\| \\
& \leq\left(1-\beta_{n}\right)\left\|x_{n}-q\right\|+\beta_{n}\left\|T x_{n}-q\right\| \\
& \leq\left(1-\beta_{n}\right)\left\|x_{n}-q\right\|+\beta_{n} \delta\left\|x_{n}-q\right\|
\end{aligned}
$$

Using (2.4) in (2.3) we get,

$$
\begin{aligned}
\left\|y_{n}-q\right\| & \leq \delta^{2}\left[\left(1-\alpha_{n}\right)\left\|x_{n}-q\right\|+\alpha_{n}\left(1-\beta_{n}\right)\left\|x_{n}-q\right\|+\alpha_{n} \beta_{n} \delta\left\|x_{n}-q\right\|\right] \\
& \leq \delta^{2}\left(1-\alpha_{n}+\alpha_{n}\left(1-\beta_{n}\right)+\alpha_{n} \beta_{n} \delta\right)\left\|x_{n}-q\right\| \\
& \leq \delta^{2}\left(1-\alpha_{n} \beta_{n}(1-\delta)\right)\left\|x_{n}-q\right\|
\end{aligned}
$$

Using (2.5) in (2.2) we get,

$$
\left\|x_{n+1}-q\right\| \leq \delta^{3}\left(1-\alpha_{n} \beta_{n}(1-\delta)\right)\left\|x_{n}-q\right\|
$$

Since $0 \leq \delta<1, \alpha_{n} \in[0,1)$ and $\sum_{n=0}^{\infty} \alpha_{n}=\infty$. Hence by using lemma (1.6), we have

$$
\lim _{n \rightarrow \infty}\left\|x_{n+1}-q\right\|=0
$$

Hence the sequence $\left\{x_{n}\right\}_{n=0}^{\infty}$ converges strongly to $q$.

Corollary 2.2: (Akewe and Okeke [14]) Let $X$ be a Banach space and $T: X \rightarrow X$ be a mapping satisfying the condition

$$
\|T x-q\| \leq \delta\|x-q\|
$$

where $q \in F, x \in X$ and $0 \leq \delta<1$. Let $\left\{x_{n}\right\}_{n=0}^{\infty}$ be the sequence defined by the Picard-Mann hybrid iterative process given by (1.3). Then the sequence $\left\{x_{n}\right\}_{n=0}^{\infty}$ converges strongly to $q$. 
Remark 2.3: Theorem 2.1 gives generalization to many results in the literature by considering a wider class of contractive type operators and more general iterative process, including the results of Chidume [15], Bosede and Rhoades [16] and Akewe and Okeke [14].

Theorem 2.4: Let $X$ be a Banach space and $T: X \rightarrow X$ be a mapping satisfying the condition

$$
\|T x-q\| \leq \delta\|x-q\|
$$

where $q \in F, x \in X$ and $0 \leq \delta<1$. Let $\left\{x_{n}\right\}_{n=0}^{\infty}$ be the sequence defined by the K-iterative process given by (1.7). Then the iteration process (1.7) is T-stable.

Proof: By theorem 2.1, the sequence $\left\{x_{n}\right\}_{n=0}^{\infty}$ converges strongly to $q$. Let $\left\{u_{n}\right\}_{n=0}^{\infty},\left\{v_{n}\right\}_{n=0}^{\infty}$ and $\left\{w_{n}\right\}_{n=0}^{\infty}$ be real sequences in $X$.

Let $\varphi_{n}=\left\|u_{n+1}-T v_{n}\right\|, n=0,1,2, \cdots$, where

$$
\begin{gathered}
w_{n}=\left(1-\beta_{n}\right) u_{n}+\beta_{n} T u_{n}, \\
v_{n}=T\left(\left(1-\alpha_{n}\right) T u_{n}+\alpha_{n} T w_{n}\right), \\
u_{n+1}=T v_{n},
\end{gathered}
$$

and let $\lim _{n \rightarrow \infty} \varphi_{n}=0$.

We shall prove that $\lim _{n \rightarrow \infty} u_{n}=q$.

Now,

$$
\begin{gathered}
\left\|u_{n+1}-q\right\|=\left\|u_{n+1}-T v_{n}\right\|+\left\|T v_{n}-q\right\| \\
\quad \leq \varphi_{n}+\delta\left\|v_{n}-q\right\| \\
\left\|v_{n}-q\right\|=\left\|T\left(\left(1-\alpha_{n}\right) T u_{n}+\alpha_{n} T w_{n}\right)-q\right\| \\
\leq \delta\left\|\left(1-\alpha_{n}\right) T u_{n}+\alpha_{n} T w_{n}-q\right\| \\
\leq \delta\left\|\left(1-\alpha_{n}\right)\left(T u_{n}-q\right)+\alpha_{n}\left(T w_{n}-q\right)\right\| \\
\leq \delta\left[\left(1-\alpha_{n}\right)\left\|T u_{n}-q\right\|+\alpha_{n}\left\|T w_{n}-q\right\|\right] \\
\leq \delta\left[\left(1-\alpha_{n}\right)\left\|T u_{n}-q\right\|+\alpha_{n}\left\|T w_{n}-q\right\|\right] \\
\leq \delta^{2}\left[\left(1-\alpha_{n}\right)\left\|u_{n}-q\right\|+\alpha_{n}\left\|w_{n}-q\right\|\right]
\end{gathered}
$$

Again using (1.7) and (2.1) we get,

$$
\begin{aligned}
\left\|w_{n}-q\right\| & =\left\|\left(1-\beta_{n}\right) u_{n}+\beta_{n} T u_{n}-q\right\| \\
& \leq\left(1-\beta_{n}\right)\left\|u_{n}-q\right\|+\beta_{n}\left\|T u_{n}-q\right\| \\
& \leq\left(1-\beta_{n}\right)\left\|u_{n}-q\right\|+\beta_{n} \delta\left\|u_{n}-q\right\| \\
& \leq\left(1-\beta_{n}(1-\delta)\right)\left\|u_{n}-q\right\|
\end{aligned}
$$

Using (2.8) in (2.7) we get,

$$
\begin{aligned}
\left\|v_{n}-q\right\| & \leq \delta^{2}\left[\left(1-\alpha_{n}\right)\left\|u_{n}-q\right\|+\alpha_{n}\left(1-\beta_{n}(1-\delta)\right)\left\|u_{n}-q\right\|\right] \\
& \leq \delta^{2}\left(1-\alpha_{n} \beta_{n}(1-\delta)\right)\left\|u_{n}-q\right\|
\end{aligned}
$$

Using (2.9) in (2.6) we get,

$$
\left\|u_{n+1}-q\right\| \leq \varphi_{n}+\delta^{3}\left(1-\alpha_{n} \beta_{n}(1-\delta)\right)\left\|u_{n}-q\right\|
$$


Since $0 \leq \delta<1$ and since $0 \leq \alpha_{n}, \beta_{n} \leq 1$ we have by lemma (1.6)

$$
\lim _{n \rightarrow \infty} u_{n}=q \text {. }
$$

Conversely let $\lim _{n \rightarrow \infty} u_{n}=q$. We shall show that $\lim _{n \rightarrow \infty} \varphi_{n}=0$.

Now

$$
\begin{aligned}
\varphi_{n} & =\left\|u_{n+1}-T v_{n}\right\| \\
& \leq\left\|u_{n+1}-q\right\|+\left\|T q-T v_{n}\right\| \\
& \leq\left\|u_{n+1}-q\right\|+\delta\left\|v_{n}-q\right\|
\end{aligned}
$$

Substituting (2.9) in (2.11),

$$
\varphi_{n} \leq\left\|u_{n+1}-q\right\|+\delta^{3}\left(1-\alpha_{n} \beta_{n}(1-\delta)\right)\left\|u_{n}-q\right\|
$$

Since $\lim _{n \rightarrow \infty} u_{n}=q$, we have from (2.12) $\lim _{n \rightarrow \infty} \varphi_{n}=0$. Hence the $\mathrm{K}$-iteration scheme is T-stable.

From theorem 2.4, we have the following corollary.

Corollary 2.5: Let $X$ be a Banach space and $T: X \rightarrow X$ be a mapping satisfying the condition

$$
\|T x-q\| \leq \delta\|x-q\|,
$$

where $q \in F, x \in X$ and $0 \leq \delta<1$. Let $\left\{x_{n}\right\}_{n=0}^{\infty}$ be the sequence defined by the Picard-Mann hybrid iterative process given by (1.3). Then the iteration process (1.3) is T-stable.

Example 2.6: Let $X=[0,1]$ and consider the mapping $T x=\frac{x}{2}$. The clearly the mapping $T$ satisfies the inequality (2.1). Now $F(T)=0$. Now we claim that the K-iteration scheme (1.7) is T-stable. Let us take $\alpha_{n}=\beta_{n}=\frac{1}{2}$ and consider the sequences $x_{n}=y_{n}=z_{n}=\frac{1}{n}$. Then clearly $\lim _{n \rightarrow \infty} x_{n}=0$.

Now

$$
\begin{aligned}
& \varphi_{n}=\left\|x_{n+1}-T y_{n}\right\|=\left\|x_{n+1}-\frac{y_{n}}{2}\right\| \\
& =\left\|x_{n+1}-\frac{T\left(\left(1-\alpha_{n}\right) T x_{n}+\alpha_{n} T z_{n}\right)}{2}\right\| \\
& =\left\|x_{n+1}-\frac{\left(1-\alpha_{n}\right) T x_{n}+\alpha_{n} T z_{n}}{4}\right\| \\
& =\left\|x_{n+1}-\left(\frac{\left(1-\alpha_{n}\right) x_{n}}{8}+\frac{\alpha_{n} z_{n}}{8}\right)\right\| \\
& =\left\|x_{n+1}-\left(\frac{\left(1-\alpha_{n}\right) x_{n}}{8}+\frac{\alpha_{n}\left(1-\beta_{n}\right) x_{n}}{8}+\frac{\alpha_{n} \beta_{n} T x_{n}}{8}\right)\right\| \\
& =\left\|x_{n+1}-\left(\frac{\left(1-\alpha_{n}\right) x_{n}}{8}+\frac{\alpha_{n}\left(1-\beta_{n}\right) x_{n}}{8}+\frac{\alpha_{n} \beta_{n} x_{n}}{16}\right)\right\| \\
& =\left\|\frac{1}{n+1}-\left(\frac{1}{16 n}+\frac{1}{32 n}+\frac{1}{64 n}\right)\right\|=\left\|\frac{1}{n+1}-\frac{1}{8 n}\right\|
\end{aligned}
$$




$$
=\left\|\frac{7 n-1}{8 n(n+1)}\right\|=\left\|\frac{7-\frac{1}{n}}{8(n+1)}\right\|
$$

Taking limit $n \rightarrow \infty$ in (2.13), we have $\lim _{n \rightarrow \infty} \varphi_{n}=0$. Hence the K-iteration process is T-stable.

Now we shall prove the convergence and stability results for asymptotically quasi-nonexpansive mapping by considering the more general form of $\mathrm{K}$-iteration process as:

$$
\begin{gathered}
z_{n}=\left(1-\beta_{n}\right) x_{n}+\beta_{n} T^{n} x_{n}, \\
y_{n}=T^{n}\left(\left(1-\alpha_{n}\right) T^{n} x_{n}+\alpha_{n} T^{n} z_{n}\right), \\
x_{n+1}=T^{n} y_{n}, \text { where } n=0,1,2, \cdots,
\end{gathered}
$$

Theorem 2.7: Let $H$ be a non-empty closed convex subset of a Banach space $X$ and $T: H \rightarrow H$ be asymptotically quasi-nonexpansive mapping with real sequence $\mu_{n} \subseteq[0, \infty)$. Let $\left\{x_{n}\right\}_{n=0}^{\infty}$ be the sequence defined by the $\mathrm{K}$-iterative process given by (2.14) and satisfies the assumption that $\sum_{n=0}^{\infty} \alpha_{n} \beta_{n} \mu_{n}=\infty$. Then the sequence $\left\{x_{n}\right\}_{n=0}^{\infty}$ converges strongly to some fixed point $q$ of the mapping $T$.

Proof: From the iterative process (2.14) we have,

$$
\begin{aligned}
\left\|z_{n}-q\right\| & =\left\|\left(1-\beta_{n}\right) x_{n}+\beta_{n} T^{n} x_{n}-q\right\| \\
& \leq\left(1-\beta_{n}\right)\left\|x_{n}-q\right\|+\beta_{n}\left\|T^{n} x_{n}-q\right\| \\
& \leq\left(1-\beta_{n}\right)\left\|x_{n}-q\right\|+\beta_{n}\left(1+\mu_{n}\right)\left\|x_{n}-q\right\| \\
& \leq\left(1+\beta_{n} \mu_{n}\right)\left\|x_{n}-q\right\|
\end{aligned}
$$

and

$$
\begin{aligned}
& \left\|y_{n}-q\right\|=\left\|T^{n}\left(\left(1-\alpha_{n}\right) T^{n} x_{n}+\alpha_{n} T^{n} z_{n}\right)-q\right\| \\
& \leq\left(1+\mu_{n}\right)\left\|\left(1-\alpha_{n}\right) T^{n} x_{n}+\alpha_{n} T^{n} z_{n}-q\right\| \\
& \leq\left(1+\mu_{n}\right)\left\|\left(1-\alpha_{n}\right)\left(T^{n} x_{n}-q\right)+\alpha_{n}\left(T^{n} z_{n}-q\right)\right\| \\
& \leq\left(1+\mu_{n}\right)\left[\left(1-\alpha_{n}\right)\left\|T^{n} x_{n}-q\right\|+\alpha_{n}\left\|T^{n} z_{n}-q\right\|\right] \\
& \leq\left(1+\mu_{n}\right)\left[\left(1-\alpha_{n}\right)\left(1+\mu_{n}\right)\left\|x_{n}-q\right\|+\alpha_{n}\left(1+\mu_{n}\right)\left\|z_{n}-q\right\|\right] \\
& \leq\left(1+\mu_{n}\right)^{2}\left[\left(1-\alpha_{n}\right)\left\|x_{n}-q\right\|+\alpha_{n}\left\|z_{n}-q\right\|\right] \\
& \leq\left(1+\mu_{n}\right)^{2}\left[\left(1-\alpha_{n}\right)\left\|x_{n}-q\right\|+\alpha_{n}\left(1+\beta_{n} \mu_{n}\right)\left\|x_{n}-q\right\|\right] \\
& \leq\left(1+\mu_{n}\right)^{2}\left(1-\alpha_{n} \beta_{n} \mu_{n}\right)\left\|x_{n}-q\right\|
\end{aligned}
$$

Again using (2.14) we have,

$$
\begin{aligned}
\left\|x_{n+1}-q\right\| & \leq\left\|T^{n} y_{n}-q\right\| \\
& \leq\left(1+\mu_{n}\right)\left\|y_{n}-q\right\| \\
& \leq\left(1+\mu_{n}\right)^{3}\left(1-\alpha_{n} \beta_{n} \mu_{n}\right)\left\|x_{n}-q\right\|
\end{aligned}
$$


By repeating the above process, we have the following inequalities

$$
\begin{gathered}
\left\|x_{n+1}-q\right\| \leq\left(1+\mu_{n}\right)^{3}\left(1-\alpha_{n} \beta_{n} \mu_{n}\right)\left\|x_{n}-q\right\| \\
\left\|x_{n}-q\right\| \leq\left(1+\mu_{n-1}\right)^{3}\left(1-\alpha_{n-1} \beta_{n-1} \mu_{n-1}\right)\left\|x_{n-1}-q\right\| \\
\left\|x_{n-1}-q\right\| \leq\left(1+\mu_{n-2}\right)^{3}\left(1-\alpha_{n-2} \beta_{n-2} \mu_{n-2}\right)\left\|x_{n-2}-q\right\| \\
\cdots \\
\left\|x_{1}-q\right\| \leq\left(1+\mu_{0}\right)^{3}\left(1-\alpha_{0} \beta_{0} \mu_{0}\right)\left\|x_{0}-q\right\|
\end{gathered}
$$

So we can write,

$$
\left\|x_{n+1}-q\right\| \leq\left(1+\mu_{0}\right)^{3(n+1)}\left\|x_{0}-q\right\| \prod_{j=0}^{n}\left(1-\alpha_{j} \beta_{j} \mu_{j}\right)
$$

Since $1-x \leq \mathrm{e}^{-x}$ for all $x \in[0,1]$. Now $1-\alpha_{j} \beta_{j} \mu_{j}<1$, so we can write,

$$
\begin{aligned}
\left\|x_{n+1}-q\right\| & \leq\left(1+\mu_{0}\right)^{3(n+1)}\left\|x_{0}-q\right\| \mathrm{e}^{-\left(1-\alpha_{j} \beta_{j} \mu_{j}\right)} \\
& \leq\left(1+\mu_{0}\right)^{3(n+1)}\left\|x_{0}-q\right\| \mathrm{e}^{-\sum_{j=0}^{n} \alpha_{j} \beta_{j} \mu_{j}}
\end{aligned}
$$

Taking limit $n \rightarrow \infty$ in (2.18), we have $\lim _{n \rightarrow \infty}\left\|x_{n}-q\right\|=0$, that is the sequence $\left\{x_{n}\right\}_{n=0}^{\infty}$ converges strongly to fixed point $q$ of the mapping $T$.

Theorem 2.8: Let $H$ be a non-empty closed convex subset of a Banach space $X$ and $T: H \rightarrow H$ be asymptotically quasi-nonexpansive mapping with real sequence $\mu_{n} \subseteq[0, \infty)$. Let $\left\{x_{n}\right\}_{n=0}^{\infty}$ be the sequence defined by the K-iterative process given by (2.14) and satisfies the assumption that $\sum_{n=0}^{\infty} \alpha_{n} \beta_{n} \mu_{n}=\infty$. Then the iterative process (2.14) is T-stable.

Proof: Let $\left\{u_{n}\right\}_{n=0}^{\infty} \subset X$ be any arbitrary sequence. Let the sequence generated by the iterative process $(2.14)$ is $x_{n+1}=f\left(T, x_{n}\right)$ converging to the fixed point $q$.

Let $\varphi_{n}=\left\|u_{n+1}-f\left(T, x_{n}\right)\right\|$.

We shall prove that $\lim _{n \rightarrow \infty} \varphi_{n}=0$ if and only if $\lim _{n \rightarrow \infty} u_{n}=q$.

First suppose $\lim _{n \rightarrow \infty} \varphi_{n}=0$. Now we have

$$
\begin{aligned}
\left\|u_{n+1}-q\right\| & =\left\|u_{n+1}-f\left(T, u_{n}\right)\right\|+\left\|f\left(T, u_{n}\right)-q\right\| \\
& =\varphi_{n}+\left\|T^{n}\left(T^{n}\left(1-\beta_{n}\right) T^{n} u_{n}+\beta_{n} T^{n}\left(\left(1-\alpha_{n}\right) u_{n}+\alpha_{n} T^{n} u_{n}\right)\right)-q\right\| \\
& \leq \varphi_{n}+\left(1+\mu_{n}\right)^{3}\left(1-\alpha_{n} \beta_{n} \mu_{n}\right)\left\|x_{n}-q\right\|
\end{aligned}
$$

where $\alpha_{n}, \beta_{n} \in[0,1], \lim _{n \rightarrow \infty} \varphi_{n}=0$ and $\lim _{n \rightarrow \infty} \mu_{n}=0$.

Now using (2.19) together with lemma (1.5), we have $\lim _{n \rightarrow \infty}\left\|u_{n}-q\right\|=0$ that is $\lim _{n \rightarrow \infty} u_{n}=q$.

Conversely let $\lim _{n \rightarrow \infty} u_{n}=q$. we have

$$
\begin{aligned}
\varphi_{n} & =\left\|u_{n+1}-f\left(T, u_{n}\right)\right\| \\
& \leq\left\|u_{n+1}-q\right\|+\left\|f\left(T, u_{n}\right)-q\right\| \\
& \leq\left\|u_{n+1}-q\right\|+\left(1+\mu_{n}\right)^{3}\left(1-\alpha_{n} \beta_{n} \mu_{n}\right)\left\|u_{n}-q\right\|
\end{aligned}
$$

Taking limit $n \rightarrow \infty$ both sides of (6) we have $\lim _{n \rightarrow \infty} \varphi_{n}=0$. Hence (2.14) is T-stable.

Now we shall prove the convergence results for mean non-expansive mapping 
by modifying the $\mathrm{K}$-iteration process for two mappings as:

$$
\begin{gathered}
z_{n}=\left(1-\beta_{n}\right) x_{n}+\beta_{n} S x_{n}, \\
y_{n}=T\left(\left(1-\alpha_{n}\right) S x_{n}+\alpha_{n} T z_{n}\right), \\
x_{n+1}=T y_{n}, \text { where } n=0,1,2, \cdots,
\end{gathered}
$$

Lemma 2.9: Let $H$ be a non-empty closed convex subset of a Banach space $X$ and $S, T: H \rightarrow H$ be two mean non-expansive mapping such that $F=F(T) \cap F(S) \neq \phi$. Let $\left\{x_{n}\right\}_{n=0}^{\infty}$ be the sequence defined by the K-iterative process given by (2.20). Then $\lim _{n \rightarrow \infty}\left\|x_{n}-q\right\|$ exists for some $q \in F$.

Proof: We have

$$
\begin{aligned}
& \left\|z_{n}-q\right\|=\left\|\left(1-\beta_{n}\right) x_{n}+\beta_{n} S x_{n}-q\right\| \\
& \leq\left(1-\beta_{n}\right)\left\|x_{n}-q\right\|+\beta_{n}\left\|S x_{n}-q\right\| \\
& \leq\left(1-\beta_{n}\right)\left\|x_{n}-q\right\|+\beta_{n}\left(a_{1}\left\|x_{n}-q\right\|+b_{1}\left\|x_{n}-q\right\|\right) \\
& \leq\left(1-\beta_{n}\right)\left\|x_{n}-q\right\|+\beta_{n}\left(a_{1}+b_{1}\right)\left\|x_{n}-q\right\| \\
& \leq\left\|x_{n}-q\right\|
\end{aligned}
$$

Again using (2.20) and (2.21)

$$
\begin{aligned}
\left\|y_{n}-q\right\| & =\left\|T\left(\left(1-\alpha_{n}\right) S x_{n}+\alpha_{n} T z_{n}\right)-q\right\| \\
& \leq a_{2}\left\|\left(\left(1-\alpha_{n}\right) S x_{n}+\alpha_{n} T z_{n}\right)-q\right\|+b_{2}\left\|\left(\left(1-\alpha_{n}\right) S x_{n}+\alpha_{n} T z_{n}\right)-q\right\| \\
& \leq\left(a_{2}+b_{2}\right)\left\|\left(\left(1-\alpha_{n}\right) S x_{n}+\alpha_{n} T z_{n}\right)-q\right\| \\
& \leq\left(1-\alpha_{n}\right)\left\|S x_{n}-q\right\|+\alpha_{n}\left\|T z_{n}-q\right\| \\
\leq & \left(1-\alpha_{n}\right)\left(a_{1}\left\|x_{n}-q\right\|+b_{1}\left\|x_{n}-q\right\|\right)+\alpha_{n}\left(a_{2}\left\|z_{n}-q\right\|+b_{2}\left\|z_{n}-q\right\|\right) \\
\leq & \left(1-\alpha_{n}\right)\left(a_{1}+b_{1}\right)\left\|x_{n}-q\right\|+\alpha_{n}\left(a_{2}+b_{2}\right)\left\|z_{n}-q\right\| \\
\leq & \left(1-\alpha_{n}\right)\left\|x_{n}-q\right\|+\alpha_{n}\left\|z_{n}-q\right\| \\
\leq & \left\|x_{n}-q\right\|
\end{aligned}
$$

Again using (2.20) and (2.22)

$$
\begin{aligned}
\left\|x_{n+1}-q\right\| & \leq\left\|T y_{n}-q\right\| \\
& \leq a_{2}\left\|y_{n}-q\right\|+b_{2}\left\|y_{n}-q\right\| \\
& \leq\left(a_{2}+b_{2}\right)\left\|y_{n}-q\right\| \\
& \leq\left\|y_{n}-q\right\| \\
& \leq\left\|x_{n}-q\right\|
\end{aligned}
$$

This shows that $\left\{\left\|x_{n}-q\right\|\right\}$ is non-increasing and bounded sequence for $q \in F$. Hence $\lim _{n \rightarrow \infty}\left\|x_{n}-q\right\|$ exists.

Lemma 2.10: Let $H$ be a non-empty closed convex subset of a Banach space $X$ and $S, T: H \rightarrow H$ be two mean non-expansive mapping such that $F=F(T) \cap F(S) \neq \phi$. Let $\left\{x_{n}\right\}_{n=0}^{\infty}$ be the sequence defined by the K-iterative process given by (2.20). Also consider that

$\lim _{n \rightarrow \infty}\left\|S x_{n}-q\right\|=\lim _{n \rightarrow \infty}\left\|T x_{n}-q\right\|=0$ for some $q \in F$. Then

$\lim _{n \rightarrow \infty}\left\|T x_{n}-x_{n}\right\|=0$. 
Proof: Let $q \in F$. In lemma (2.9) we have proved the existence of

$$
\lim _{n \rightarrow \infty}\left\|x_{n}-q\right\| \text {. Let } \lim _{n \rightarrow \infty}\left\|x_{n}-q\right\|=c .
$$

W.L.O.G. let $c>0$.

Now from (2.20) and (2.24) we have,

$$
\underset{n \rightarrow \infty}{\limsup }\left\|z_{n}-q\right\| \leq \limsup _{n \rightarrow \infty}\left\|x_{n}-q\right\|=c
$$

Now

$$
\begin{aligned}
\left\|S x_{n}-q\right\| & \leq a_{1}\left\|x_{n}-q\right\|+b_{1}\left\|x_{n}-q\right\| \\
& \leq\left(a_{1}+b_{1}\right)\left\|x_{n}-q\right\| \leq\left\|x_{n}-q\right\|
\end{aligned}
$$

Implies that $\limsup _{n \rightarrow \infty}\left\|S x_{n}-q\right\| \leq \limsup _{n \rightarrow \infty}\left\|x_{n}-q\right\|=c$

Now

$$
\begin{aligned}
&\left\|x_{n+1}-q\right\| \leq\left\|T y_{n}-q\right\| \leq a_{2}\left\|y_{n}-q\right\|+b_{2}\left\|y_{n}-q\right\| \\
& \leq\left(a_{2}+b_{2}\right)\left\|y_{n}-q\right\| \leq\left\|y_{n}-q\right\| \\
& \leq\left\|T\left(\left(1-\alpha_{n}\right) S x_{n}+\alpha_{n} T z_{n}\right)-q\right\| \\
& \leq a_{2}\left\|\left(\left(1-\alpha_{n}\right) S x_{n}+\alpha_{n} T z_{n}\right)-q\right\|+b_{2}\left\|\left(\left(1-\alpha_{n}\right) S x_{n}+\alpha_{n} T z_{n}\right)-q\right\| \\
& \quad \leq\left(a_{2}+b_{2}\right)\left\|\left(\left(1-\alpha_{n}\right) S x_{n}+\alpha_{n} T z_{n}\right)-q\right\| \\
& \leq\left(1-\alpha_{n}\right)\left\|S x_{n}-q\right\|+\alpha_{n}\left\|T z_{n}-q\right\| \\
& \leq\left(1-\alpha_{n}\right)\left(a_{1}\left\|x_{n}-q\right\|+b_{1}\left\|x_{n}-q\right\|\right)+\alpha_{n}\left(a_{2}\left\|z_{n}-q\right\|+b_{2}\left\|z_{n}-q\right\|\right) \\
& \leq\left(1-\alpha_{n}\right)\left(a_{1}+b_{1}\right)\left\|x_{n}-q\right\|+\alpha_{n}\left(a_{2}+b_{2}\right)\left\|z_{n}-q\right\| \\
& \leq\left(1-\alpha_{n}\right)\left\|x_{n}-q\right\|+\alpha_{n}\left\|z_{n}-q\right\| \\
& \leq\left\|x_{n}-q\right\|-\alpha_{n}\left\|x_{n}-q\right\|+\alpha_{n}\left\|z_{n}-q\right\| \\
& \Rightarrow \frac{\left\|x_{n+1}-q\right\|-\left\|x_{n}-q\right\|}{\alpha_{n}}=\left\|z_{n}-q\right\|-\left\|x_{n}-q\right\|
\end{aligned}
$$

and hence

$$
\begin{gathered}
\left\|x_{n+1}-q\right\|-\left\|x_{n}-q\right\| \leq \frac{\left\|x_{n+1}-q\right\|-\left\|x_{n}-q\right\|}{\alpha_{n}}=\left\|z_{n}-q\right\|-\left\|x_{n}-q\right\| \\
\text { which implies that }\left\|x_{n+1}-q\right\| \leq\left\|z_{n}-q\right\|
\end{gathered}
$$

Taking limit inferior in (2.27) we obtain

$$
c \leq \liminf _{n \rightarrow \infty}\left\|z_{n}-q\right\|
$$

From (2.20) and (2.28) we have

$$
\begin{aligned}
c & =\lim _{n \rightarrow \infty}\left\|z_{n}-q\right\| \\
& =\lim _{n \rightarrow \infty}\left\|\left(1-\beta_{n}\right) x_{n}+\beta_{n} S x_{n}-q\right\| \\
& =\lim _{n \rightarrow \infty}\left\|\beta_{n}\left(S x_{n}-q\right)+\left(1-\beta_{n}\right)\left(x_{n}-q\right)\right\|
\end{aligned}
$$

Now from (2.24), (2.26), (2.29) and lemma (1.7), we have $\lim _{n \rightarrow \infty}\left\|S x_{n}-x_{n}\right\|=0$. Now, 


$$
\begin{gathered}
\left\|T x_{n}-q\right\| \leq a_{2}\left\|x_{n}-q\right\|+b_{2}\left\|x_{n}-q\right\| \leq\left\|x_{n}-q\right\| \\
\Rightarrow \underset{n \rightarrow \infty}{\limsup }\left\|T x_{n}-q\right\| \leq \limsup _{n \rightarrow \infty}\left\|x_{n}-q\right\| \leq c
\end{gathered}
$$

Using the conditions of the lemma in (2.30), we can write

$$
C=\lim _{n \rightarrow \infty}\left\|\beta_{n}\left(T x_{n}-q\right)+\left(1-\beta_{n}\right)\left(x_{n}-q\right)\right\|
$$

Using (2.24), (2.30), (2.31) along with the lemma (1.7), we have

$$
\lim _{n \rightarrow \infty}\left\|T x_{n}-x_{n}\right\|=0 \text {. }
$$

Theorem 2.11: Let $H$ be a non-empty closed convex subset of a Banach space $X$ satisfying Opial's condition and $S, T$ and $\left\{x_{n}\right\}_{n=0}^{\infty}$ be same as defined in the lemma (2.10). Then the sequence $\left\{x_{n}\right\}_{n=0}^{\infty}$ converges weakly to some $q \in F$.

Proof: From lemma (2.10) we have, $\lim _{n \rightarrow \infty}\left\|T x_{n}-x_{n}\right\|=0$.

Since $X$ is uniformly convex and hence it is reflexive so there exists a subsequence $\left\{x_{n_{m}}\right\}$ of $\left\{x_{n}\right\}$ such that $\left\{x_{n_{m}}\right\}$ converges weakly to some $q_{1} \in F$. Since $H$ is closed so $q_{1} \in H$. Now we claim the weak convergence of $\left\{x_{n}\right\}$ to $q_{1}$. Let it is not true, then there exists a subsequence of $\left\{x_{n_{i}}\right\}$ of $\left\{x_{n}\right\}$ which converges weakly to $q_{2}$ and let $q_{1} \neq q_{2}$. Also $q_{2} \in F$. Now from lemma (2.9) $\lim _{n \rightarrow \infty}\left\|x_{n}-q_{1}\right\|$ and $\lim _{n \rightarrow \infty}\left\|x_{n}-q_{2}\right\|$ both exist. Using Opial's condition we have,

$$
\begin{aligned}
\lim _{n \rightarrow \infty}\left\|x_{n}-q_{1}\right\| & \leq \lim _{n \rightarrow \infty}\left\|x_{n_{m}}-q_{1}\right\|<\lim _{n \rightarrow \infty}\left\|x_{n_{m}}-q_{2}\right\| \\
& =\lim _{n \rightarrow \infty}\left\|x_{n}-q_{2}\right\|=\lim _{n \rightarrow \infty}\left\|x_{n_{i}}-q_{2}\right\| \\
& <\lim _{n \rightarrow \infty}\left\|x_{n_{i}}-q_{1}\right\| \leq \lim _{n \rightarrow \infty}\left\|x_{n}-q_{1}\right\|
\end{aligned}
$$

This is a contradiction, so we must have $q_{1}=q_{2}$. Thus the sequence $\left\{x_{n}\right\}_{n=0}^{\infty}$ converges weakly to some $q \in F$.

Theorem 2.12: Let $H$ be a non-empty closed compact subset of a Banach space $X$ and $S, T$ and $\left\{x_{n}\right\}_{n=0}^{\infty}$ be same as defined in the lemma (2.10). Then the sequence $\left\{x_{n}\right\}_{n=0}^{\infty}$ converges strongly to some $q \in F$.

Proof: Since $H$ is compact and hence it is sequentially compact. So there exists a subsequence $\left\{x_{n_{i}}\right\}$ of $\left\{x_{n}\right\}$ which converges to $q \in H$.

Now

$$
\begin{aligned}
\left\|x_{n_{i}}-T q\right\| & =\left\|x_{n_{i}}-T x_{n_{i}}\right\|+\left\|T x_{n_{i}}-T q\right\| \\
& \leq\left\|x_{n_{i}}-T x_{n_{i}}\right\|+a_{2}\left\|x_{n_{i}}-q\right\|+b_{2}\left\|x_{n_{i}}-q\right\| \\
& \leq\left\|x_{n_{i}}-T x_{n_{i}}\right\|+\left\|x_{n_{i}}-q\right\|
\end{aligned}
$$

Taking limit $n \rightarrow \infty$ in (2.32) we have, $T q=q$ that is $q \in F$. We have earlier proved that $\lim _{n \rightarrow \infty}\left\|x_{n}-q\right\|$ exists for $q \in F$. Hence the sequence $\left\{x_{n}\right\}_{n=0}^{\infty}$ converges strongly to some $q \in F$.

In [8] it is proves that the K-iteration process converges faster than Picard-S, Thakur-New and Vatan two-step iterative process. Now we shall compare the rate of convergence the $\mathrm{K}$-iteration process defined in [8] and our new modified $\mathrm{K}$-iteration process for two mappings. 
Table 1. Iterative values of $\mathrm{K}$-iteration process and Modified $\mathrm{K}$-iteration process.

\begin{tabular}{ccc}
\hline & K-iteration & Modified K-iteration \\
\hline$x_{0}$ & 2.25 & 2.25 \\
$x_{1}$ & 2.030273437500000 & 2.013360362386860 \\
$x_{2}$ & 2.003665924072266 & 2.000717402289730 \\
$x_{3}$ & 2.000443920493126 & 2.000038531785984 \\
$x_{4}$ & 2.000053755997215 & 2.000002069576723 \\
$x_{5}$ & 2.000006509515288 & 2.000000111158901 \\
$x_{6}$ & 2.000000788261617 & 2.000000005970444 \\
$x_{7}$ & 2.000000095453555 & 2.000000000320678 \\
$x_{8}$ & 2.000000011558829 & 2.000000000017224 \\
$x_{9}$ & 2.000000001399702 & 2.000000000000925 \\
$x_{10}$ & 2.000000000169495 & 2.0000000000000050 \\
$x_{11}$ & 2.000000000020525 & 2.0000000000000003 \\
$x_{12}$ & 2.000000000002486 & 2.000000000000000 \\
$x_{13}$ & 2.000000000000301 & 2.0000000000000000 \\
$x_{14}$ & 2.000000000000036 & 2.000000000000000 \\
$x_{15}$ & 2.000000000000004 & 2.000000000000000 \\
$x_{16}$ & 2.000000000000000 & 2.000000000000000 \\
& &
\end{tabular}

Example 2.13: Let $S, T:[0,3] \rightarrow[0,3]$ be two mappings defined by $T(x)=\frac{x+2}{2}$ and $s(x)=(x+2)^{\frac{1}{2}}$. Let $\alpha_{n}, \beta_{n}$ be the sequences defined by $\alpha_{n}=\beta_{n}=\frac{1}{4}$. Let the initial approximation be $x_{0}=2.25$. Clearly $S, T$ has unique common fixed point 2 . The convergence pattern of K-iteration process and modified $\mathrm{K}$-iteration process is shown in Table 1.

Clearly we can conclude from Table 1, that the modified K-iteration process has better rate of convergence than the k-iteration process.

\section{Conflicts of Interest}

The authors declare no conflicts of interest regarding the publication of this paper.

\section{References}

[1] Mann, W.R. (1953) Mean Value Methods in Iteration. Proceedings of the American Mathematical Society, 4, 506-510. https://doi.org/10.1090/S0002-9939-1953-0054846-3

[2] Khan, S.H. (2013) A Picard-Mann Hybrid Iterative Process. Fixed Point Theory and Applications, 2013, 69. https://doi.org/10.1186/1687-1812-2013-69

[3] Ishikawa, S. (1974) Fixed Points by a New Iteration Method. Proceedings of the American Mathematical Society, 44, 147-150. 
https://doi.org/10.1090/S0002-9939-1974-0336469-5

[4] Gursoy, F. and Karakaya, V. (2014) A Picard-S Hybrid Type Iteration Method for Solving a Differential Equation with Retared Arguments. 1-16.

[5] Karakaya, V., Bouzara, N.E.H., Dogan, K. and Atalan, Y. (2015) On Different Results for a New Two Step Iteration Method under Weak Contraction Mapping in Banach Spaces. 1-10. arXiv:1507.00200v1

[6] Thakur, B.S., Thakur, D. and Postolache, M. (2016) A New Iterative Scheme for Numerical Reckoning Fixed Points of Suzuki's Generalized Non-Expansive Mappings. Applied Mathematics and Computation, 275, 147-155. https://doi.org/10.1016/j.amc.2015.11.065

[7] Opial, Z. (1967) Weak Convergence of the Sequence of Successive Approximations for Non-Expansive Mappings. Bulletin of the American Mathematical Society, 73, 595-597. https://doi.org/10.1090/S0002-9904-1967-11761-0

[8] Hussain, N., Ullah, K. and Arshad, M. (2018) Fixed Point Approximation of Suzuki Generalized Non-Expansive Mapping via New Faster Iterative Process. arxiv $1802.09888 \mathrm{v}$

[9] Zhang, S.S. (1975) About Fixed Point Theory for Mean Non Expansive Mappings in Banach Spaces. Journal of Sichuan University, 2, 67-78.

[10] Harder, A.M. (1987) Fixed Point Theory and Stability Results for Fixed Point Iteration Procedure. University of Missouri-Rolla, Missouri.

[11] Weng, X. (1991) Fixed Point Iteration for Local Strictly Pseudo-Contractive Mapping. Proceedings of the American Mathematical Society, 113, 727-731. https://doi.org/10.1090/S0002-9939-1991-1086345-8

[12] Berinde, V. (2002) On the Stability of Some Fixed Point Procedures. Buletinul ştiințific al Universitatii Baia Mare, Seria B, Fascicola matematică-informatică, 18, 7-14.

[13] Sahu, J. (1991) Weak and Strong Convergence to Fixed Points of Asymptotically Non-Expansive Mappings. Bulletin of the Australian Mathematical Society, 43, 153-159. https://doi.org/10.1017/S0004972700028884

[14] Akewe, H. and Okeke, G.A. (2015) Convergence and Stability Theorems for the Picard-Mann Hybrid Iterative Scheme for a General Class of Contractive-Like Operators. Fixed Point Theory and Applications, 2015, 66.

[15] Chidume, C.E. (2014) Strong Convergence and Stability of Picard Iteration Sequence for General Class of Contractive-Type Mappings. Fixed Point Theory and Applications, 2014, 233. https://doi.org/10.1186/1687-1812-2014-233

[16] Bosede, A.O. and Rhoades, B.E. (2010) Stability of Picard and Mann Iteration for a General Class of Functions. Journal of Advanced Mathematical Studies, 3, 23. 